consumed on the heaviest drinking day. In addition, ordinary least squares regression analyses were used, modelling the proportion non-drinking as the independent variable, and the proportion binge/mean units as the dependent variable.

Results Rates of non-drinking increased from 18\% [95\% CI $16 \%$ to $22 \%$ ] in 2005 to $29 \%$ [25-33\%] in 2015 (test for trend; $\mathrm{p}<0.001)$, largely attributable to increases in lifetime abstention. Not drinking in the past week increased from $35 \%$ [32-39\%] to 50\% [45-55\%] $(\mathrm{p}<0.001)$. Significant linear increases in non-drinking were found among most subgroups including healthier sub-groups (non-smokers, those with high physical activity and good mental health), north and south regions, in full-time education, and employed. Among white participants, non-drinking increased from 14\% [12$17 \%$ ] in 2005 to $20 \%$ [17-24\%] in 2015. No significant increases in non-drinking were found among smokers, ethnic minorities and those with poor mental health. At the population-level, a one percentage point increase in non-drinking, predicted a 0.22 reduction in mean alcohol units consumed [95\% CI -0.32 to 0.12 ], and a 1.06 percentage point decrease in the proportion binge drinking [95\% CI 1.56 to 0.54]

Conclusion Increases in non-drinking were found with variables less commonly associated with non-drinking, suggesting this behaviour may be becoming more normative. This trend is to be welcomed from a public health standpoint and should be capitalised on going forward. Drinking and smoking continue to cluster and could be targeted in tandem. Future research should explore attitudes towards alcohol among young people.

\section{OP77 CAN MENTAL HEALTH COMPETENCE BUFFER AGAINST THE HIGHER RISK OF SMOKING INITIATION AMONG TEENAGERS WITH PARENTS WHO SMOKE? FINDINGS FROM THE UK MILLENNIUM COHORT STUDY}

${ }^{1,2} \mathrm{~A}$ Pearce* ${ }^{2} \mathrm{E}$ Rougeaux, ${ }^{3} \mathrm{~J}$ Deighton, ${ }^{2} \mathrm{RM}$ Viner, ${ }^{2} \mathrm{C}$ Law, ${ }^{2} \mathrm{~S}$ Hope. ${ }^{1} \mathrm{MRC} / \mathrm{CSO}$ Social and Public Health Sciences Unit, University of Glasgow, Glasgow, UK; ${ }^{2}$ Population, Policy and Practice Programme, UCL Great Ormond Street Institute of Child Health, London, UK; ${ }^{3}$ Evidence Based Practice Unit, UCL and the Anna Freud Centre, London, UK

\subsection{6/jech-2018-SSMabstracts.76}

Background Most smokers initiate before the age of eighteen, and smoking in adolescence is strongly influenced by parental smoking habits. Despite this, few studies have examined factors which may protect teenagers against the risk of having a smoking parent. We investigated whether skills-based components of positive mental health in childhood (Mental Health Competence, MHC) modified the association between parent and teenager smoking, using contemporary data from the UKrepresentative Millennium Cohort Study ( $\sim 18000$ children, born 2000-2002; analytic sample: $n=10,133)$.

Methods Cohort members (CMs) reported whether they had ever smoked cigarettes or e-cigarettes at 14 years(y). A dichotomised variable indicated whether one or both parents reported their own tobacco use when CMs were 11 y. A four-class latent measure of MHC captured learning skills and prosocial behaviours at $11 \mathrm{y}$ : High, High-Moderate, Moderate, Low.

We examined effect modification in two ways. First, we compared risk differences (RD; estimated using binary regression) for $\mathrm{CM}$ smoking according to parental smoking, across levels of MHC. Second, we estimated RDs for CM smoking according to combinations of parental smoking and MHC. Confounding by socio-economic and demographic characteristics and parent's mental health was adjusted for. Survey weights accounted for sample design and attrition; multiple imputation addressed item missingness.

Results Similar proportions of CMs had ever smoked cigarettes $(17 \%)$ and e-cigarettes (18\%), although overlap was moderate (40\% who had smoked either had smoked both). CMs were more likely to have ever smoked cigarettes if at least one parent smoked (RD: 16\%[13-184]) (baseline: no parents who smoke[11\%]). This elevated risk was observed across all levels of MHC, but was greatest for Low MHC (RD: 21\%[11-31] (RDs in other MHC groups: 7\%-12\%). When combining parental smoking and MHC (baseline: no smoking parent, high MHC), those with Low MHC and a smoking parent had a RD of $28 \%(20-36)$. This was higher than the sum of RDs in CMs with Low MHC but a non-smoking parent (7\%[114]) and with High MHC but a smoking parent (11\% [715]). Thus, Low MHC carried an excess risk. There was little evidence of effect modification by Moderate or High-Moderate MHC. Results were similar for e-cigarettes.

Conclusion The association between parent smoking and teenage smoking initiation was considerably stronger in those with Low MHC in contemporary, UK-representative data. These results require replication in other populations, and at older ages (examining progression to regular smoking), but imply that MHC improvement before transition to secondary school holds potential to buffer against an important smoking risk factor.

The views expressed are those of the authors and not necessarily those of the funders.

\section{Physical activity}

\section{OP78 ARE QUALITY OF LIFE AND FAMILY EXPENDITURE ON PHYSICAL ACTIVITY ASSOCIATED WITH PHYSICAL ACTIVITY IN 2-4-YEAR-OLD CHILDREN?}

${ }^{1} \mathrm{~L}$ Tinner*, ${ }^{1} \mathrm{R}$ Kipping, ${ }^{2} \mathrm{~J}$ White, ${ }^{3} \mathrm{R}$ Jago, ${ }^{1} \mathrm{C}$ Metcalfe, ${ }^{1} \mathrm{~W}$ Hollingworth. ${ }^{1}$ Population Health Sciences, University of Bristol, Bristol, UK; ${ }^{2}$ Centre for Trials Research, University of Cardiff, UK; ${ }^{3}$ School for Policy Studies, University of Bristol, UK

\subsection{6/jech-2018-SSMabstracts.77}

Background Despite the well-described health benefits associated with physical activity, many children do not engage in the recommended level of physical activity. To inform public health interventions, there is a need to determine factors associated with physical activity in children. We examined the extent to which the Pediatric Quality of Life Inventory (PedsQL) and family expenditure on physical activity were associated with minutes spent physically active and in moderate-to-vigorous physical activity (MVPA) per day in young children.

Methods Cross-sectional study with a sample of 81 children aged 2-4 years in the South West of England, taking part in the Nutrition and Physical Activity Self-Assessment for Child Care (NAPSACC UK) feasibility randomized controlled trial. We ran descriptive statistics, along with Student t-tests to determine differences by gender, age and parental education and compare physical activity on nursery and non-nursery days. The associations between physical activity, PedsQL scores (physical and psychosocial) and family expenditure on physical 